\title{
Expression of Heat Shock Protein 105 in Cutaneous Squamous Cell Carcinoma: Correlation with Clinicopathological Characteristics
}

\author{
Meng Jia \\ Feng-Zeng $\mathrm{Li}$ \\ Qian Ye \\ Ke-Jun Chen \\ Sheng Fang (D)
}

Department of Dermatology, The First Affiliated Hospital of Chongqing Medical University, Chongqing, People's Republic of China
Correspondence: Sheng Fang Department of Dermatology, The First Affiliated Hospital of Chongqing Medical University, No. I Youyi Road, Yuzhong

District, Chongqing, 400016, People's Republic of China

Email fangshengderm@163.com
Background: Heat shock proteins (HSPs), a group of heat stress proteins, are characterized by highly conserved properties. Malignant transformation is a cellular stress, and the expression of HSPs may be affected during this process. Heat shock protein 105 (HSP105) is a protective protein that has long been observed in many cancer types, but little attention has been given to cutaneous squamous cell carcinoma (CSCC). As such, the objectives of this study were to observe the expression of HSP105 on CSCC and evaluate its correlation with clinicopathological characteristics.

Methods: This retrospective study enrolled 60 patients with CSCC. The patients' clinical data, including sex, age, tumor location, tumor type, and degree of pathological differentiation, were collected. The expression of HSP105 was measured by Western blot and immunohistochemical staining.

Results: HSP105 expression was decreased in CSCC (HSCORE $=0.65(0.30,1.98)$ ) compared with normal skin (HSCORE=2.20 (1.50, 2.80)) $(\mathrm{P}<0.001)$. These results were consistent with the Western blot analysis. HSP105 immunostaining of Bowen disease $($ HSCORE $=1.28(1.08,2.40))$ revealed higher expression than in verrucous carcinoma $(\operatorname{HSCORE}=0.30(0.23,0.85))$, keratoacanthoma $(\operatorname{HSCORE}=0.53(0.29,0.93))$ and acantholytic squamous cell carcinoma (HSCORE $=0.53(0.41,0.68)(\mathrm{P}<0.01))$. Poorly differentiated CSCC showed significantly higher expression of HSP105.

Conclusion: Our study reveals for the first time that the expression of HSP105 is decreased in CSCC. We suggest that the molecular mechanisms underlying the differential expression of HSP deserve a more rigorous future study, the results of which might explain its role in carcinogenesis and its potential as a target for selective tumor therapy.

Keywords: heat shock protein 105, cutaneous, squamous cell carcinoma, expression

\section{Introduction}

The incidence of cutaneous squamous cell carcinoma (CSCC), one of the most common skin cancers, is second only to basal cell carcinoma (BCC). Although it is recognized that UV exposure is a preventable risk factor for CSCC, from 1992 to 2012, the number of keratinocyte carcinomas, including CSCC and BCC, in the US population increased by $100 \%{ }^{1}$

Heat shock proteins (HSPs), major components of the cellular proteoprotection system, are involved in tumor cell proliferation and the prevention of apoptosis. HSPs are characterized by diverse intracellular localization and functional differentiation. When organisms are stimulated by external conditions, HSPs participate 
in the folding, assembly and transportation of newly synthesized proteins and prevent denaturation or nonspecific aggregation of improperly folded proteins. ${ }^{2}$ HSP105, a member of the HSP110 family, is a mammalian stress protein with high molecular weight that is released in response to a variety of stress factors, such as infection, tumors, heat and ischemia. ${ }^{3,4}$

Previous research has shown that HSP105 is overexpressed in a variety of human tumors. ${ }^{5}$ Nooshin et al showed the association between HSP27 and HSP105 and prognosis in tongue SCC, and HSP105 is a promising agent for cancer immunotherapy. ${ }^{6}$ Souzana et al studied the expression of HSP105 in oral SCCs, and their results suggested that the overexpression of HSP105 may be a prognostic factor for malignancy in oral SCC. ${ }^{7}$ Muchemwa et al reported that tumors overexpressing HSP105 are ideal candidates for vaccination with HSP105-derived peptides or DNA. ${ }^{8}$ However, an association between the level of HSP105 expression and CSCC has thus far been lacking. We therefore aimed to study HSP105 expression in CSCC and evaluate its correlation with clinicopathological characteristics.

\section{Materials and Methods}

Ethics approval was granted by the First Affiliated Hospital of Chongqing Medical University \& Ethics unit (No 2021-004). A retrospective review was conducted of patients seen at the First Affiliated Hospital of Chongqing Medical University, Chongqing, China, between January 2016 and October 2020. Patients were eligible to participate if they were diagnosed with CSCC. Clinical data were collected from medical records. The First Affiliated Hospital of Chongqing Medical University Ethics Committee approved the collection of pathological wax blocks and patient clinical information for medical research purposes. The patients' written informed consent was given to present their clinical pictures with no identifiable information. Sixty specimens of CSCC were analyzed. We divided the tumors by location into the exposed site (head, face, neck, V-neck, outer forearms and dorsum of the hand) group and the nonexposed site group.

\section{Western Blot}

Tissue samples for Western blot analysis were frozen in liquid nitrogen soon after excision and kept at $-80^{\circ} \mathrm{C}$. The specimens consisted of CSCC and normal skin; patients provided informed consent before surgery. The biopsy samples were lysed, and the cytoplasmic fractions were separated. The cytoplasmic fractions were dissolved in RIPA buffer supplemented with phosphatase inhibitor (Roche). Protein aliquots were loaded into each well of an SDS-PAGE gel for isolation. For better detection of protein expression levels, the proteins in the SDS-PAGE gel were transferred to a nitrocellulose membrane (Millipore) and the membrane was blocked in $5 \%(\mathrm{w} / \mathrm{v})$ bovine serum albumin for 1 hour, followed by incubation with HSP105 antibody (Abcam HSP105/HSP110; item number 109624) overnight. The membranes were then rinsed and incubated with the secondary antibody for 1 hour at room temperature. After rinsing the membranes thoroughly with PBST, signals were detected using an Odyssey fluorescence scanner (LI-COR Bioscience, Inc., Lincoln, NE, USA).

\section{Immunohistochemistry}

Paraffin sections $(4 \mu \mathrm{m})$ were dewaxed by dipping in xylene for 10 minutes and were subsequently hydrated with different alcohol gradients. The sections were soaked in sodium citrate antigen repair solution, boiled for 20 minutes at $100{ }^{\circ} \mathrm{C}$, and then cooled to room temperature. To inhibit endogenous peroxidase reaction in the tissue, the tissue was treated with $3 \%$ hydrogen peroxide for 10 minutes and then blocked with 3\% BSA at room temperature for 1 hour. These sections were placed in a wet room and incubated with HSP105 antibody (Abcam HSP105/ HSP110; item number 109624) at $4^{\circ} \mathrm{C}$ overnight. After several washes with PBST (Triton X-100 washing solution), the secondary antibody was added and the sections were incubated at room temperature for 40 minutes. After washing three times, sections were treated with $D A B$ visualization reagent, and nuclei were counterstained with hematoxylin. Finally, sections were subjected to alcohol gradient dehydration, xylene transparency treatment, neutral resin sealing, and air-drying. We then observed the sections with a pathological scanner.

\section{Evaluation of HSPI05 Expression}

For the immunohistochemical evaluation, the histochemical score (HSCORE) was used to evaluate the intensity and percentage of stained tumors by two senior skin pathologists. The HSCORE $=\sum \operatorname{Pi}(\mathrm{i}+1)$, $\mathrm{i}$ is the intensity of staining with a value of 0,1 , or 2 ( 0 , no or light coloring; 1, medium-light brownish-yellow; 2, dark brownish-yellow), $\mathrm{Pi}$ is the percentage of stained tumor cells varying from $0 \%$ to $100 \%(0.0-100)$. HSCOREs ranged from a minimum of 0 in unstained cases to 
a maximum of 3.0 in all cases with the highest intensity of tumor cell staining. Because of the abnormal distribution in our data, the median (M) and upper and lower quartiles (P25, P75) were used to describe the HSCORE.

\section{Statistical Analysis}

All statistical analyses were performed with SPSS software for Windows (Version 26.0). HSP105 expression was analyzed using the nonparametric Mann-Whitney and Kruskal-Wallis tests. $\mathrm{P}$ values were judged as significant if they were less than 0.05 .

\section{Results}

\section{General Data}

A total of 60 patients with CSCC were identified. The general data are presented in Table 1. Out of all of the patients with CSCC, men (46.67\%) were affected less commonly than women $(53.33 \%)$. The average age was 69.25 years old (range 39-97 years). There were 31 invasive CSCC cases with 14 well differentiated and 17 poorly differentiated cases. In addition, other variants included Bowen disease ( 8 cases), verrucous carcinoma ( 8 cases), keratoacanthoma ( 7 cases) and acantholytic squamous cell carcinoma (6 cases). The lesions of 33 patients with CSCC were located on nonexposed sites, while 27 patients had tumors located at exposed sites.

\section{Expression of HSPI05 in CSCC and}

\section{Normal Epidermis}

The positive expression of HSP105 in both the epidermis of normal skin and the CSCC is located in the cytoplasm and visualized as light yellow to brown, and the positive cells are dotted, focal or diffusely distributed. HSP105 was highly expressed in the spiny cells of the normal epidermis but not in the basal cells, while the pattern of expression of HSP105 in CSCC tissues was opposite of that in the normal epidermis, with weak expression in the spiny cells and positive expression in the basal cells. Differences in the expression of HSP105 between the normal epidermis (HSCORE=2.20 (1.50, 2.80)) and $\mathrm{CSCC}(\mathrm{HSCORE}=0.65$ (0.30, 1.98)) were remarkable $(\mathrm{P}<0.001)$ (Figure 1$)$. The results were consistent with those obtained by Western blot analysis, which revealed lower HSP105 expression in CSCC $(\mathrm{P}<0.05)$ (Figure 2).

\section{The Relationship Between HSPI05 and the Clinical Features}

The correlations between HSP105 immunostaining and tumor clinicopathological features (tumor location, degrees of differentiation, clinical variants, Breslow thickness) are summarized in Table 2. In different clinical

Table I Clinicopathological Features of CSCC

\begin{tabular}{|c|c|c|c|c|c|c|}
\hline \multirow[t]{2}{*}{ Variables } & \multirow[t]{2}{*}{ Total } & \multicolumn{2}{|c|}{ Gender } & \multicolumn{3}{|c|}{ Age } \\
\hline & & Male & Female & Mean & Median & Range \\
\hline \multicolumn{7}{|l|}{ Differentiation } \\
\hline Well differentiated & 14 & 5 & 9 & $67.5 y$ & $62.5 y$ & $45 \sim 89 y$ \\
\hline Poorly differentiated & 17 & 7 & 10 & $60.8 y$ & $58.5 y$ & $50 \sim 87 y$ \\
\hline \multicolumn{7}{|l|}{ Variants } \\
\hline Invasive CSCC & 31 & 12 & 19 & $60.3 y$ & $61.5 y$ & $45 \sim 89 y$ \\
\hline Bowen disease & 8 & 3 & 5 & $67.8 y$ & $68.5 y$ & $59 \sim 77 y$ \\
\hline Verrucous carcinoma & 8 & 4 & 4 & $65.7 y$ & $67.6 y$ & $42 \sim 88 y$ \\
\hline Keratoacanthoma & 7 & 5 & 2 & $63 y$ & $63 y$ & $38 \sim 85 y$ \\
\hline ASCC & 6 & 2 & 4 & 72.ly & $71.5 y$ & $64 \sim 86 y$ \\
\hline \multicolumn{7}{|l|}{ Sites } \\
\hline Exposure sites & 27 & 15 & 12 & $67.8 y$ & $69 y$ & $39 \sim 81 y$ \\
\hline Non-exposure sites & 33 & 13 & 20 & $73.6 y$ & $74 y$ & $44 \sim 97 y$ \\
\hline \multicolumn{7}{|l|}{ Breslow thickness } \\
\hline Grade $\mathrm{I}(<3 \mathrm{~mm})$ & 12 & 5 & 7 & $62.5 y$ & $60 y$ & $48 \sim 89 y$ \\
\hline Grade II(3 6mm) & 33 & 14 & 19 & $72.2 y$ & $77 y$ & $47 \sim 86 y$ \\
\hline Grade III $(>6 \mathrm{~mm})$ & 15 & 9 & 6 & $69.8 y$ & $73 y$ & $4 \mid \sim 92 y$ \\
\hline
\end{tabular}

Abbreviations: CSCC, cutaneous squamous cell carcinoma; ASCC, acantholytic squamous cell carcinoma. 

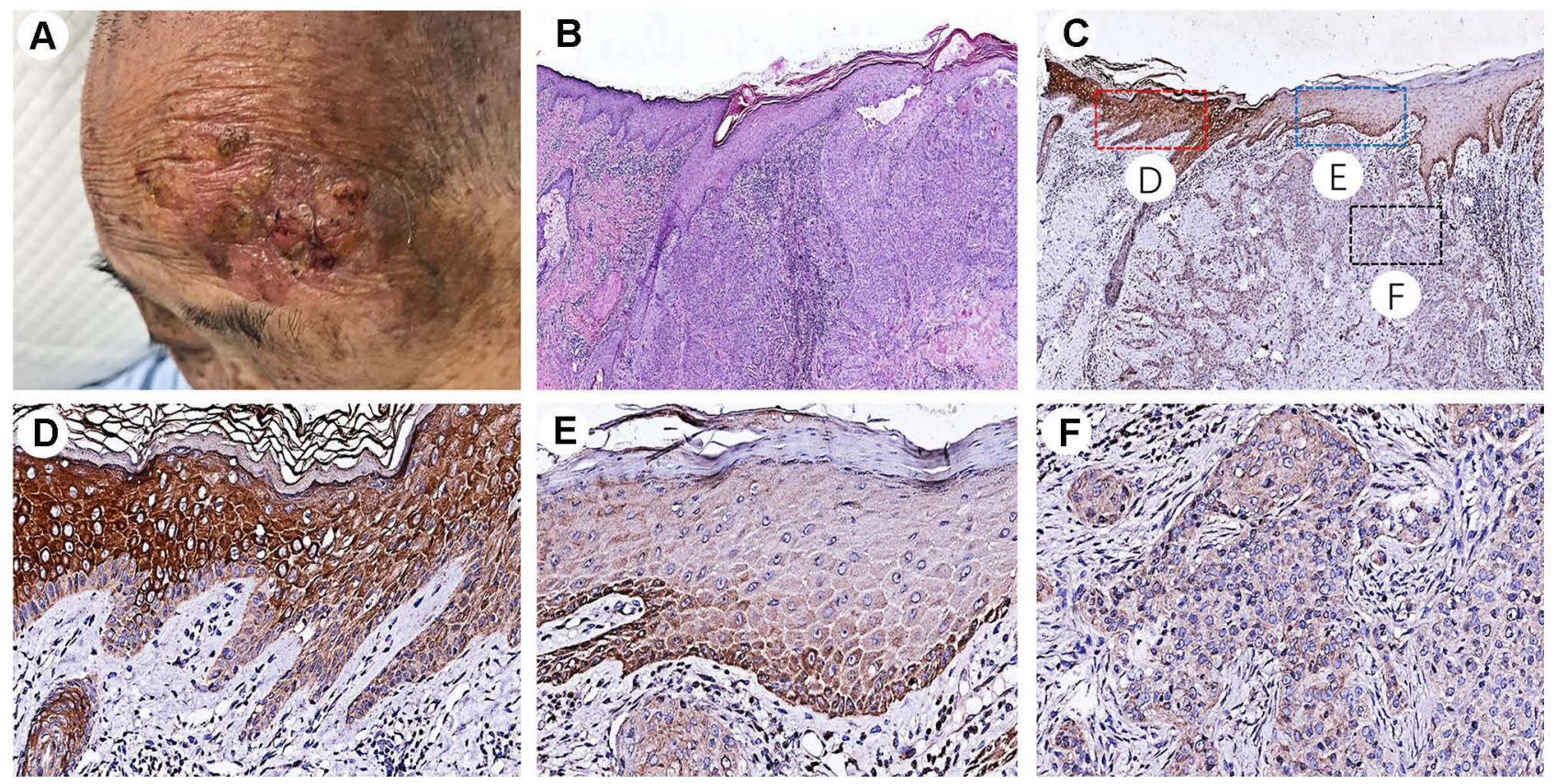

Figure I (A, B) Clinical and histopathological findings of CSCC (HE×20). (C) Immunohistochemical staining of HSP I05 ( $\times 20)$. (D) HSPI05 is highly expressed in spiny cells but not in basal cells of normal epidermis next to CSCC $(\times 200)$. (E) The expression of HSPI05 in CSCC was lower in spiny cells and positive expression was found in basal cells. (F) HSPI05 is expressed at low levels in CSCC in the dermis $(\times 200)$.

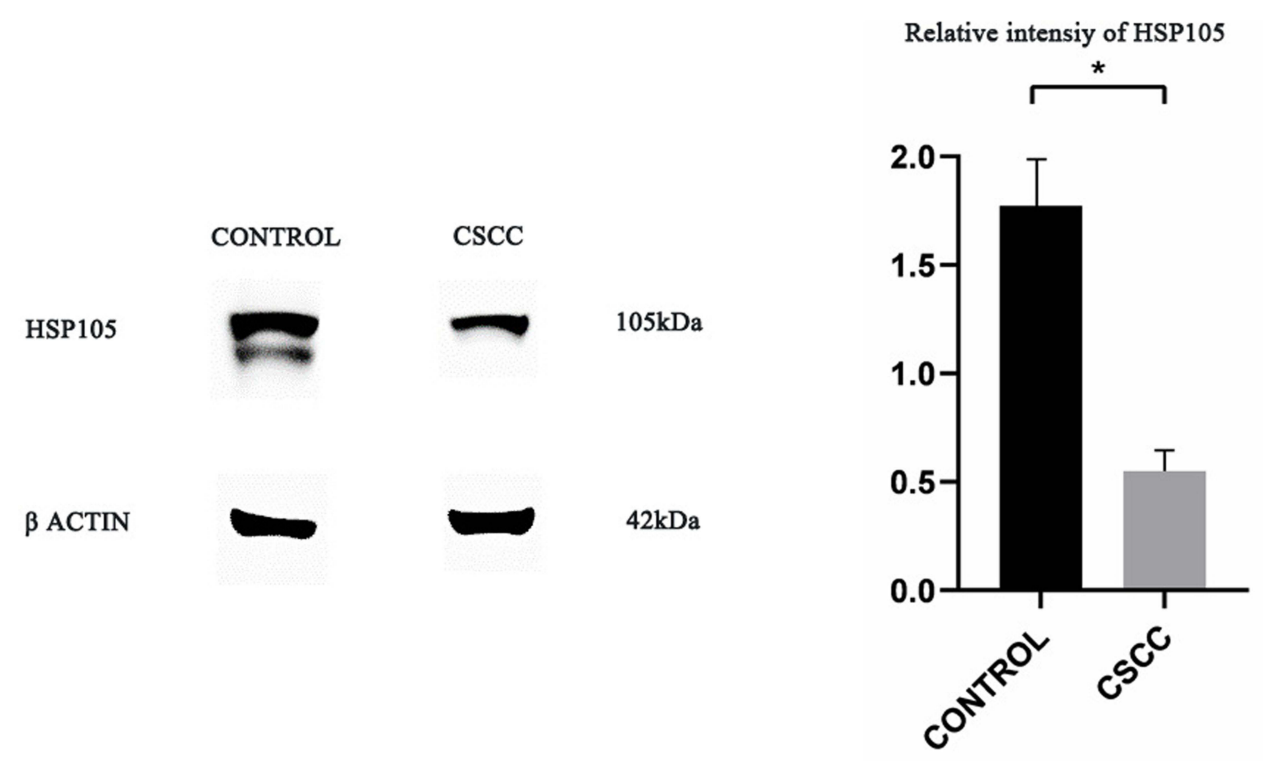

Figure 2 Western blot analysis of HSPI05 expression in CSCC and controls (normal skin). The comparison is shown in the column graph. Values represent the mean \pm SD. $*, \mathrm{P}<0.05$.

variants, HSP105 immunostaining of Bowen disease (squamous cell carcinoma in situ) (HSCORE=1.28 (1.08, 2.40)) was higher than that of verrucous carcinoma $(\mathrm{HSCORE}=0.30 \quad(0.23,0.85)), \quad$ keratoacanthoma $(\operatorname{HSCORE}=0.53(0.29,0.93))$ and acantholytic squamous cell carcinoma (ASCC) (HSCORE $=0.53(0.41,0.68)$ )
$(\mathrm{P}<0.01)$ (Figure 3). However, there was no significant difference between verrucous carcinoma, keratoacanthoma and ASCC in this study. Although the expression of HSP105 was higher in lesions of exposed sites (especially on the lips) than those at nonexposed sites, no statistically significant difference was found $(\mathrm{P}>0.05)$. 
Table 2 HSPI05 Expression in Different Clinicopathological Features of CSCC

\begin{tabular}{|c|c|c|c|c|c|c|}
\hline \multirow[t]{2}{*}{ Variables } & \multirow{2}{*}{$\begin{array}{l}\text { Median Number of HSP I } 05 \\
\text { Positive Cells }\end{array}$} & \multicolumn{3}{|c|}{ Intensity of HSP I 05 Expression (\%) } & \multirow{2}{*}{$\begin{array}{l}\text { HSCORE for HSPI } 05 \\
\text { Evaluation } \\
\text { M (P25,P75) }\end{array}$} & \multirow[t]{2}{*}{ P-value } \\
\hline & & - & + & ++ & & \\
\hline \multicolumn{7}{|l|}{ Differentiation } \\
\hline Well differentiated & 45 & $53.3(n=8)$ & $46.7(n=7)$ & - & $0.60(0.35,1.00)$ & $\mathrm{P}<0.05$ \\
\hline Poorly differentiated & 55 & $18.7(n=3)$ & $43.8(n=7)$ & $37.5(n=6)$ & $1.30(0.78,1.84)$ & \\
\hline \multicolumn{7}{|l|}{ Variants } \\
\hline Bowen & 58 & - & $50.0(n=4)$ & $50.0(n=4)$ & $1.28(1.08,2.40)$ & $\mathrm{P}<0.01$ \\
\hline Verrucous carcinoma & 30 & $42.9(n=3)$ & $57.1 \quad(n=4)$ & - & $0.30(0.23,0.85)$ & \\
\hline Keratoacanthoma & 40 & $50.0(n=4)$ & $50.0(n=4)$ & - & $0.53(0.29,0.93)$ & \\
\hline ASCC & 35 & $33.3(n=2)$ & $66.7(n=4)$ & - & $0.53(0.41,0.68)$ & \\
\hline \multicolumn{7}{|l|}{ Sites } \\
\hline Exposure sites & 35 & $29.7(n=8)$ & $63.0(n=17)$ & $7.30(n=2)$ & $0.70(0.20,1.35)$ & $\mathrm{P}=0.840$ \\
\hline Non-exposure sites & 45 & $27.2(n=9)$ & $57.6(n=19)$ & $15.2(n=5)$ & $0.90(0.10,1.40)$ & \\
\hline \multicolumn{7}{|l|}{ Breslow thickness } \\
\hline Grade I(<3mm) & 46 & $25.0(n=3)$ & $58.3(n=7)$ & $16.7(n=2)$ & $\mathrm{I} .00(0.18, \mathrm{I} .3 \mathrm{I})$ & $\mathrm{P}=0.90 \mathrm{I}$ \\
\hline Grade II(3 6mm) & 35 & $33.3(n=11)$ & $57.6(n=19)$ & $9.1(n=3)$ & $0.70(0.20,1.40)$ & \\
\hline Grade III(>6mm) & 65 & $40.0(n=6)$ & $53.3(n=8)$ & $6.7(n=1)$ & $0.80(0.20,1.20)$ & \\
\hline
\end{tabular}

Abbreviations: CSCC, cutaneous squamous cell carcinoma; ASCC, acantholytic squamous cell carcinoma.

\section{The Relationship Between HSPI05 and Pathological Features}

When the expression of HSP105 was analyzed with respect to the degrees of differentiation, a strong correlation was observed. The well-differentiated cells in CSCC, especially those near the corneal beads and the stratum corneum, did not express HSP105. In contrast, poorly differentiated CSCC cells showed higher expression HSP105 expression. Differences between the expression of HSP105 and the degree of differentiation were remarkable $(\mathrm{P}<0.05)$ (Figure 4).

HSP105 expression was compared among the three groups of grade I (Breslow thickness $<3 \mathrm{~mm}$ ), grade II (Breslow thickness between 3 and $6 \mathrm{~mm}$ ), and grade III (Breslow thickness $>6 \mathrm{~mm}$ ) tumors, but there was no significant correlation between the expression of HSP105 and Breslow thickness $(\mathrm{P}=0.901)$.

\section{Discussion}

The tumor mass of CSCC is comprised of a heterogeneous population of malignant keratinocytes at various stages of differentiation. Its development may be related to excessive sun exposure or trauma and chronic infection, or it may be transformed as a result of actinic keratosis, seborrheic keratosis and other precancerous lesions. ${ }^{9}$ Although surgery is the main treatment for CSCC, the outcomes of the currently available treatments for advanced CSCC have been disappointing. Inhibition of key molecular targets in multiple pro-survival/growth signals could be a better approach for the treatment of CSCC.

Stress inducibility is a characteristic shared by most HSPs, as they play a substantial protective role when cells are stimulated by stressful conditions. ${ }^{10}$ The presence of HSP72 and HSP105 in the exosomes of sera from tumor patients, but not healthy people, suggests their significant value for tumor prediction. ${ }^{11}$ Previous studies have found that HSP105 is overexpressed in a variety of human cancers, including colorectal, pancreatic, thyroid, esophageal, and breast carcinoma. ${ }^{5,12}$ Serum samples from pancreatic and colorectal cancer patients have also been previously used to define HSP105 expression by serum recombinant expression clones. ${ }^{13}$ An HSP105-pulsed bone marrow DC vaccine is an effective immunotherapy for tumors that appear spontaneously due to inactivation of tumor suppressor genes. ${ }^{14}$ This finding further illustrates the immunogenic properties of HSP105 in cancer.

HSP105 has two isoforms, $\alpha$ and $\beta$, the former being produced under physiological and pathological conditions, while the latter is specifically produced in response to heat stress. ${ }^{15}$ p53 is a tumor suppressor gene that functions to help cells with defective gene repair pathways. The tumor suppressor p53 maintains genomic stability by regulating a variety of cell functions (including cell cycle arrest, 

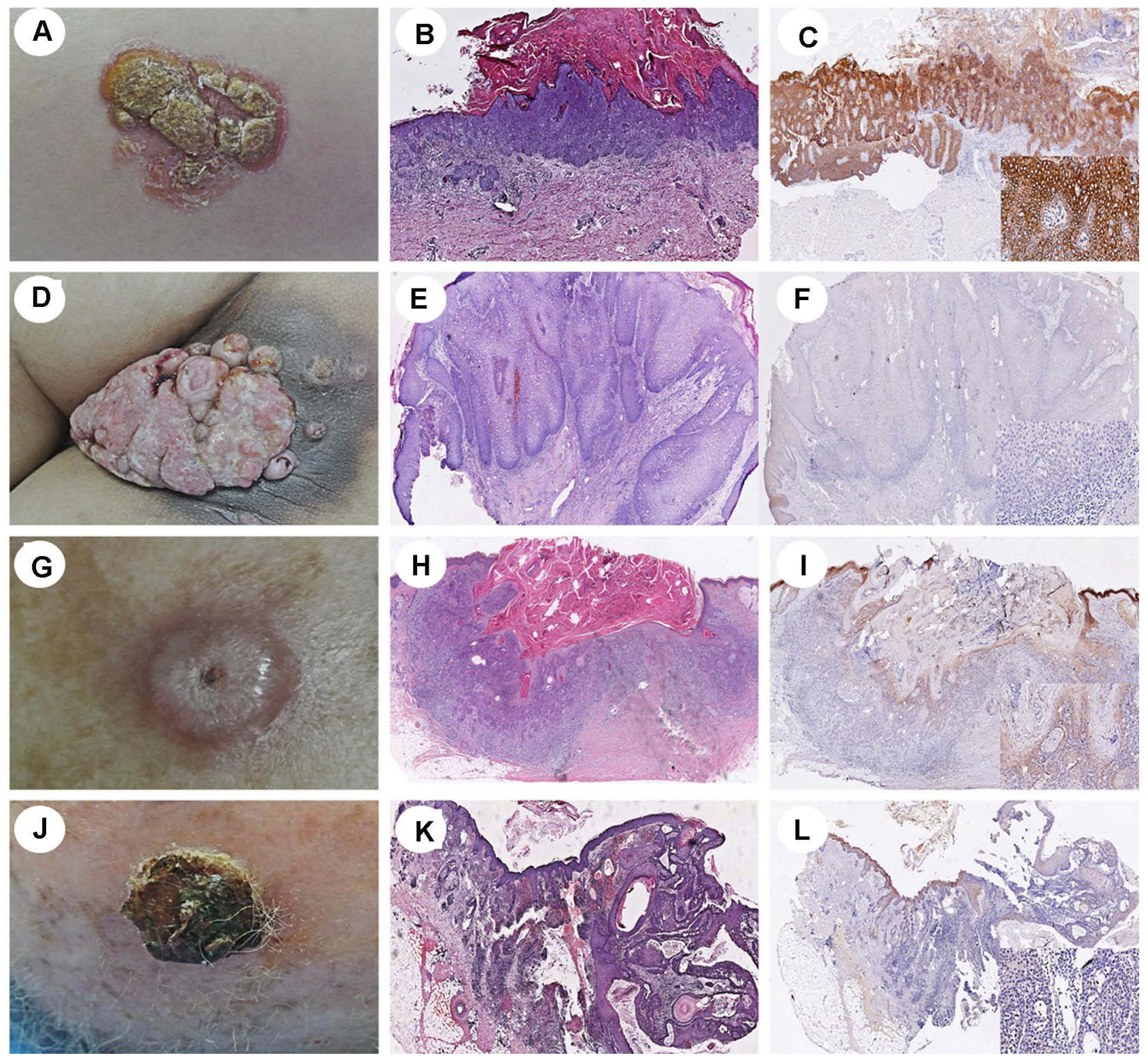

Figure 3 Clinical and histopathological appearances of Bowen (A, B; HEx|0), verrucous carcinoma (D, E; HEx|0), keratoacanthoma (G, H; HExI0) and acantholytic SCC (J, K; HExI0). HSPI05 is highly expressed in Bowen, while shows a trend of lower expression in verrucous carcinoma, keratoacanthoma and acantholytic SCC (C, F, I, L; $\mathrm{IHC} \times 10)$.

apoptosis, aging and metabolic homeostasis). The loss of p53 leads to abnormal cell proliferation and cell death, and ultimately leads to tumor formation. ${ }^{16}$ HSP105 has an inhibitory effect on p53-induced apoptosis after binding to p53 in the cytoplasm. Heat stimulation of HSP105 separates it from $\mathrm{p} 53$ and transports it to the nucleus, leading to apoptosis. ${ }^{17}$

Fernández-Guarino et al reported that increased levels of cytoplasmic HSP70 is a potential new biomarker for early infiltration of CSCC caused by actinic keratosis. ${ }^{18}$ Singh et al showed that topical 17AAG may be useful for the prevention of UV radiation (UVR)-induced inflammation and that CSCC develops in either UVR-exposed or organ transplant populations. ${ }^{19}$ We initially investigated the expression of three heat shock proteins in CSCC, HSP70, HSP90, HSP105, with HSP70 and HSP90 overexpressed in both normal human skin and CSCC. Only HSP105 was reduced in CSCC, whereas it was overexpressed in normal human skin. Therefore, we hypothesize that HSP105 is a more relevant tumor-associated antigen in CSCC than HSP70 and HSP90. Previous studies have revealed that HSP105 is overexpressed in CSCC and oral 

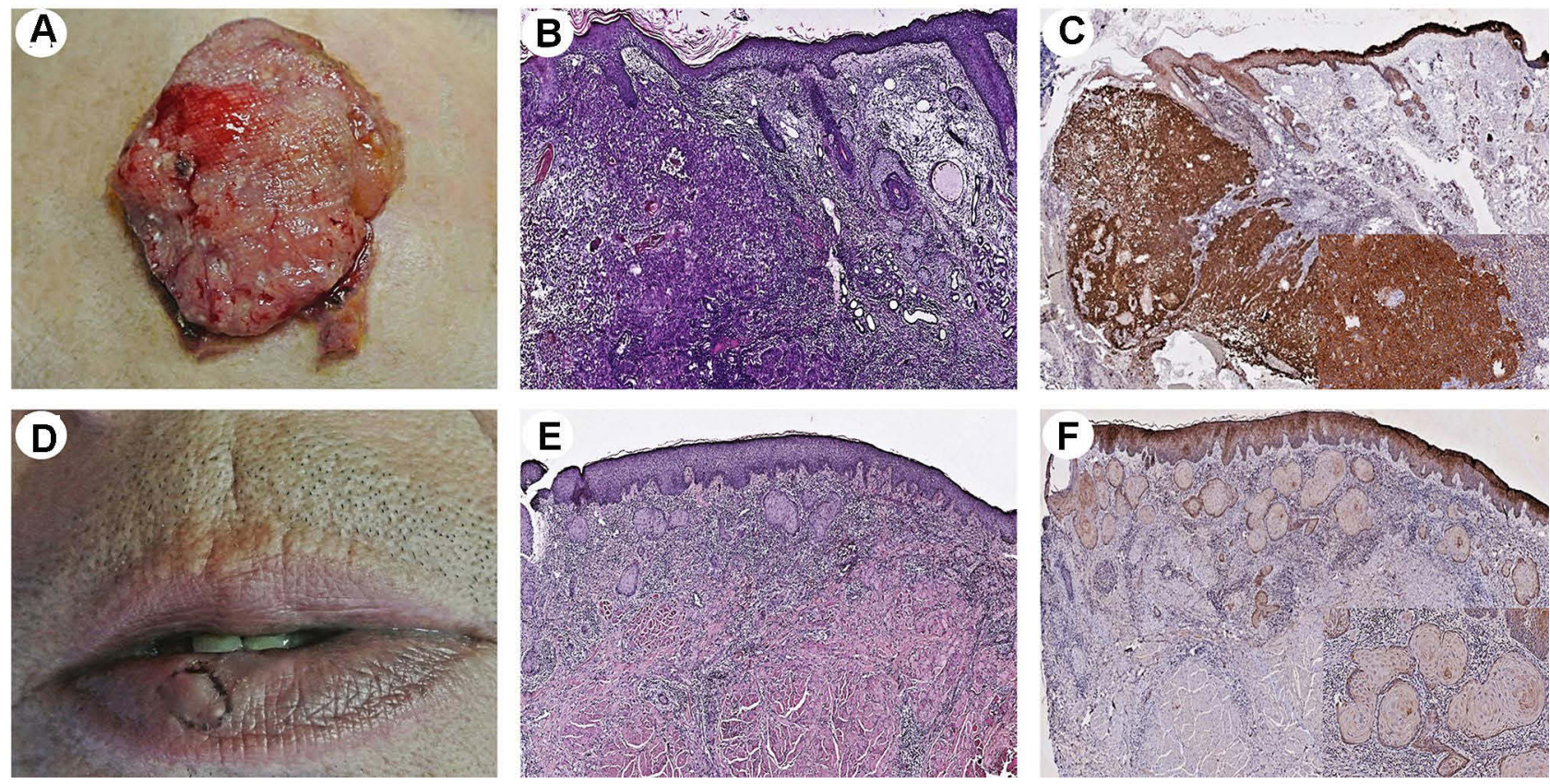

Figure 4 Clinical picture of poorly differentiated CSCC (A) and well-differentiated CSCC (D). Histopathological findings of poorly differentiated CSCC (B; HEx20) and well-differentiated CSCC (E; HE×20). HSPI05 is more highly expressed in poorly differentiated CSCC $(\mathbf{C}$; IHC $\times 20)$ than in well-differentiated CSCC (F; IHC $\times 20)$.

SCC. ${ }^{7,8}$ The present work shows, for the first time to our knowledge, a lower expression of HSP105 in CSCC compared to the normal epidermis. These findings suggest that HSP105 may play different roles in different tumors and merit further investigation.

For cancer resection specimens, staging is feasible and necessary to indicate the malignancy of the tumor and the direction of treatment. Poorly differentiated CSCC is much more malignant than well-differentiated CSCC. In the current study, we revealed that poorly differentiated CSCC showed higher HSP105 expression. A progressive increase in HSP105 expression from well differentiated to poorly differentiated CSCC indicates of an association between this marker and lesion stage. Previous studies have reported that high expression of HSP105 in squamous cell carcinoma of the tongue and lung adenocarcinoma was associated with disease progression and/or poor prognosis. ${ }^{6,20}$ Another study suggested that increased expression of HSP105 in oral squamous cell carcinoma could be an unfavorable factor for malignancy. ${ }^{7}$ These findings are consistent with our results. We believe that poorly differentiated CSCC cells exhibit a significantly faster rate of cell aggregation and proliferation with a higher proliferation index, which may be related to higher expression of HSP105, which may function to help cancer cells maintain functional integrity during tumor proliferation and prevent apoptosis.

Analysis of 60 CSCC samples focusing on different subtypes demonstrated that HSP105 was relatively highly expressed in Bowen disease but showed a trend of lower expression in verrucous carcinoma, keratoacanthoma and ASCC. Bowen disease, also known as squamous cell carcinoma in situ, is characterized by marked cell atypia and poor differentiation, while verrucous carcinoma is a slow growing, well demarcated, exophytic variant of squamous cell carcinoma with a characteristic verrucous presentation. Keratoacanthoma is a controversial epithelial hyperplasia. Some researchers believe that it represents a type of well-differentiated variant of squamous cell carcinoma, while others regard it as a benign tumor or borderline malignant tumor. $^{21}$ ASCC is a histologically unique variant of squamous cell carcinoma, and some have suggested that it may be more aggressive than conventional squamous cell carcinoma. ${ }^{22}$ According to Yoshifuku et al, 8-OHdG expression was significantly increased in Bowen lesions compared to surrounding nonlesional tissue, SCC lesions and healthy controls. ${ }^{23}$ The biomarker $8-\mathrm{OHdG}$ has been pivotal for measuring the effect of endogenous oxidative damage to DNA and is a factor involved in the initiation and promotion of carcinogenesis. Therefore, we speculate 
that the high expression of HSP105 in Bowen disease might be related to DNA damage or marked cell atypia and poor differentiation. The low expression in verrucous carcinoma, keratoacanthoma and ASCC may be consistent with the high level of differentiation.

Interestingly, although the expression of HSP105 was higher in lesions located at exposed sites (especially on the lips) than those located in nonexposed sites, we did not identify a significant difference between the expression of HSP105 and various sites of CSCC lesions. Excessive UVR exposure is the most relevant etiology for the development of CSCC, and UVR initiates photocarcinogenesis by directly damaging DNA. ${ }^{19}$ Tawanda et al believed that UV exposure is the main influencing factor for the occurrence of CSCC, which is inconsistent with the findings of this study. ${ }^{24} \mathrm{We}$ speculate that the reason for this is that the onset of CSCC is not only caused by UVR but may also be related to other factors. A study revealed that, in colorectal cancer, positive expression of HSP105 is associated with clinical stage and positive lymph node involvement, suggesting that HSP105 plays an important role in tumor metastasis progression. ${ }^{25}$ However, according to another study in which it was revealed that tumors with lymphovascular invasion and lymph node metastasis exhibit a tendency for lower HSP105 scores, low HSP105 expression may be associated with lymphovascular invasion and/or metastasis independent of the depth of tumor invasion, which is consistent with our results. ${ }^{26}$ There was no relationship between Breslow thickness and HSP105 expression in our study.

In conclusion, we examined the level of HSP105 expression in CSCC and evaluated the relationship between HSP105 and the clinicopathological characteristics of CSCC. HSP105 is overexpressed in various human tumors; in contrast, the novel finding of this study is that the expression of HSP105 showed trend to be lower in CSCC compared to the normal epidermis. Higher HSP105 expression is associated with Bowen disease but lower expression was observed in verrucous carcinoma, keratoacanthoma and ASCC. We also found that poorly differentiated CSCC showed higher expression with remarkable differences, but there was no significant difference related to tumor location or Breslow thickness.

\section{Ethical Approval}

Ethical approval was given by the Medical Ethics Committee, the First Affiliated Hospital of Chongqing Medical University (Reference number: 2021-004). This study was conducted in accordance with the Declaration of Helsinki.

\section{Funding}

There is no funding to report.

\section{Disclosure}

The authors declare no conflict of interest related to this study.

\section{References}

1. Rosenberg AR, Tabacchi M, Ngo KH, et al. Skin cancer precursor immunotherapy for squamous cell carcinoma prevention. JCI Insight. 2019;4(6):e125476.

2. Scieglinska S, Krawczyk D, Sojka DR, Gogler-Pigłowska A. Heat shock proteins in the physiology and pathophysiology of epidermal keratinocytes. Cell Stress Chaperones. 2019;24(6):1027-1044.

3. Morris SD. Heat shock proteins and the skin. Clin Exp Dermatol. 2002;27(3):220-224.

4. Xue JH, Fukuyama H, Nonoguchi K, et al. Induction of Apg-1, a member of the heat shock protein 110 family, following transient forebrain ischemia in the rat brain. Biochem Biophys Res Commun. 1998;247(3):796-801.

5. Kai M, Nakatsura T, Egami H, Senju S, Nishimura Y, Ogawa M. Heat shock protein 105 is overexpressed in a variety of human tumors. Oncol Rep. 2003;10(6):1777-1782.

6. Mohtasham N, Babakoohi S, Montaser-Kouhsari L, et al. The expression of heat shock proteins 27 and 105 in squamous cell carcinoma of the tongue and relationship with clinicopathological index. Med Oral Patol Oral Cir Bucal. 2011;16(6):e730e735.

7. Arvanitidou S, Martinelli-Kläy CP, Samson J, Lobrinus JA, Dulguerov N, Lombardi T. HSP105 expression in oral squamous cell carcinoma: correlation with clinicopathological features and outcomes. J Oral Pathol Med. 2020;49(7):665-671.

8. Muchemwa FC, Nakatsura T, Ihn H, Kageshita T. Heat shock protein 105 is overexpressed in squamous cell carcinoma and extramammary Paget disease but not in basal cell carcinoma. Br J Dermatol. 2006;155(3):582-585.

9. Green AC, Olsen CM. Cutaneous squamous cell carcinoma: an epidemiological review. Br J Dermatol. 2017;177 (2):373-381.

10. Zininga T, Ramatsui L, Shonhai A. Heat shock proteins as immunomodulants. Molecules. 2018;23(11):2846.

11. Park HS, Park CH, Choi BR, et al. Expression of heat shock protein 105 and 70 in malignant melanoma and benign melanocytic nevi. J Cutan Pathol. 2009;36(5):511-516.

12. Zappasodi R, Bongarzone I, Ghedini GC, et al. Serological identification of HSP105 as a novel non-Hodgkin lymphoma therapeutic target. Blood. 2011;118(16):4421-4430.

13. Yasuda K, Nakai A, Hatayama T, Nagata K. Cloning and expression of murine high molecular mass heat shock proteins, HSP105. J Biol Chem. 1995;270(50):29718-29723.

14. Yokomine K, Nakatsura T, Senju S, et al. Regression of intestinal adenomas by vaccination with heat shock protein 105-pulsed bone marrow-derived dendritic cells in ApcMin/+ mice. Cancer Sci. 2010;99(12):1930-1935.

15. Miyazaki M, Nakatsura T, Yokomine K, et al. DNA vaccination of HSP105 leads to tumor rejection of colorectal cancer and melanoma in mice through activation of both CD4 T cells and CD8 T cells. Cancer Sci. 2005;96(10):695-705.

16. Berger C, Qian Y, Chen X. The p53-estrogen receptor loop in cancer. Curr Mol Med. 2013;13(8):1229-1240. 
17. Kumagai J, Fukuda J, Kodama H, et al. Germ cell-specific heat shock protein 105 binds to p53 in a temperature-sensitive manner in rat testis. Eur J Biochem. 2000;267(10):3073-3078.

18. Fernández-Guarino M, León JJZ, Farré AJL, et al. Cytoplasmic increase in Hsp70 protein: a potential new biomarker of early infiltration of cutaneous squamous cell carcinoma arising from actinic keratosis. Cancers. 2020;12(5):1151.

19. Singh A, Singh A, Sand JM, et al. Topically applied Hsp90 inhibitor 17AAG inhibits UVR-induced cutaneous squamous cell carcinomas. J Invest Dermatol. 2015;135(4):1098-1107.

20. Oda TM, Eiich I, et al. Prognostic significance of heat shock protein 105 in lung adenocarcinoma. Mol Med Rep. 2009;2(4):603-607.

21. Parekh V, Seykora JT. Cutaneous squamous cell carcinoma. Clin Lab Med. 2017;37(3):503-525.

22. Pyne JH, Myint E, Barr EM, Clark SP, David M, Na R. Acantholytic invasive squamous cell carcinoma: tumor diameter, invasion depth, grade of differentiation, surgical margins, perineural invasion, recurrence and death rate. J Cutan Pathol. 2017;44 (4):320-327.
23. Yoshifuku A, Fujii K, Kanekura T. Comparison of oxidative stress on DNA, protein and lipids in patients with actinic keratosis, Bowen's disease and squamous cell carcinoma. $J$ Dermatol. 2018;45 (11):1319-1323.

24. Schmitt J, Haufe E, Trautmann F, et al. Is ultraviolet exposure acquired at work the most important risk factor for cutaneous squamous cell carcinoma? Results of the population-based case-control study FB-181. Br J Dermatol. 2018;178(2):462-472.

25. Hwang TS, Han HS, Choi HK, et al. Differential, stage-dependent expression of Hsp70, Hsp110 and $\mathrm{Bcl}-2$ in colorectal cancer. $J$ Gastroenterol Hepatol. 2003;18(6):690-700.

26. Kawai T, Enomoto Y, Morikawa T, et al. High expression of heat shock protein 105 predicts a favorable prognosis for patients with urinary bladder cancer treated with radical cystectomy. Mol Clin Oncol. 2014;2(1):38-42.

\section{Publish your work in this journal}

Clinical, Cosmetic and Investigational Dermatology is an international, peer-reviewed, open access, online journal that focuses on the latest clinical and experimental research in all aspects of skin disease and cosmetic interventions. This journal is indexed on CAS.
The manuscript management system is completely online and includes a very quick and fair peer-review system, which is all easy to use. Visit http://www.dovepress.com/testimonials.php to read real quotes from published authors. 